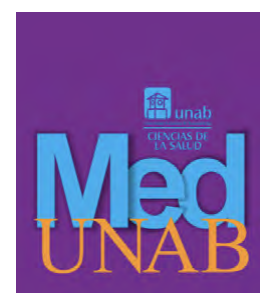

REVISTA DE LA FACULTAD

DE CIENCIAS DE LA SALUD

\title{
La toma de decisiones de gobiernos y sectores de la sociedad, intuiciones y datos en tiempos de pandemia SARS-COVID-19
}

\author{
Decision-making by Governments and Sectors of Society, Intuitions and Data in \\ Times of the SARS-COVID-19 Pandemic
}

A tomada de decisões de governos e setores da sociedade, intuições e dados em tempos de pandemia SARS-COVID-19

Uno de los cuestionamientos más recurrentes durante la pandemia ha girado alrededor de la toma de decisiones y el tipo de evidencia que las respalda. Comparada con otras crisis como la económica del 2008, que principalmente afectó al sector financiero; o el escenario de malas decisiones durante la epidemia de la gripe aviar en la década de los setenta (1), la pandemia por COVID-19 ha afectado todo el sistema socioeconómico y generando incertidumbre ha aumentado la percepción de riesgo. Esto ha hecho que se requieran tiempos de respuesta más cortos, así como una reflexión del sistema de valores de las sociedades en el proceso de toma de decisiones. En este contexto, las evidencias provienen de diferentes sectores y de diferentes ramas del conocimiento. La pregunta que razonablemente surge entonces es: ¿A quién escuchar?

La rapidez con que se deben tomar las decisiones en tiempos de pandemia requiere un ajuste al modelo de toma de decisiones basado en evidencia que demanda información con alta credibilidad y libre de sesgos. Por ejemplo, el resultado de investigación científica de calidad. Supone además que la información disponible sea el resultado de un exhaustivo análisis y una evaluación metódica, además de accesible y fácil de entender.

En la actual coyuntura estos estrictos requerimientos son casi imposibles de atender. Se ha pasado de utilizar exclusivamente información de alta credibilidad, a aceptar información razonablemente cré́ble; de priorizar la información mediante una evaluación sistemática, se ha pasado a hacer una valoración desde la subjetividad del juicio profesional y la experiencia de quienes evalúan la información disponible.

Esta transición, se ha convertido en un reto tripartito para la toma de decisiones en estos tiempos: (i) para la ciencia, la necesidad de una comunicación pronta;(ii) para los gobiernos, que requieren entender la ciencia, pero con 
la cual no están familiarizados, y (iii) para la sociedad en general, y su búsqueda de respuestas que reduzcan la incertidumbre del momento. A medida que la pandemia ha ido desarrollándose, los gobiernos, a su propio ritmo, han respondido a este reto creando equipos con variados niveles de interdisciplinariedad, incluyendo desde profesionales de salud hasta profesionales de otras disciplinas del saber y personal experto en la toma de decisiones in situ.

Países como Inglaterra y Alemania, aunque difieren en el modelo usado para abordar la pandemia, comparten una larga historia de cercanía entre la sociedad, la ciencia y la política - esta historia es mucho más corta para los países latinoamericanos-. El tiempo apremia y no hay lugar para esfuerzos aislados, tener equipos interdisciplinarios se ha convertido en el ideal administrativo de la pandemia para muchos gobiernos.

La efectividad del aporte de los equipos interdisciplinarios en la toma de decisiones depende de la información que manejen, científica o no, y del acumulado de conocimiento intuitivo que haya en el equipo sobre cómo hacer el mejor uso posible de la información disponible. El conocimiento intuitivo resulta del entrenamiento profesional y de la experiencia de trabajo en el área de experticia; este no se improvisa, ni se enseña, ni se aprende. Aunque carece de trazabilidad, es conocimiento a la mano y de rápido acceso (2), característica que no necesariamente le resta legitimidad. La intuición es una memoria acumulada, resultado del proceso de reflexión que la persona hace sobre sus propios comportamientos y la lógica de sus pensamientos. Por ejemplo, intuiciones para la toma de decisiones resultan del análisis que históricamente una persona haya hecho sobre su propio proceso de toma de decisiones (3).

El procesamiento rápido de información es necesario en situaciones donde compiten tantas variables, y en estos casos las intuiciones son una guía automática en el proceso de toma de decisiones. El error no está en usar la intuición, sino en seguir a personas con poca intuición o con intuiciones que no son el resultado de un proceso de reflexión continuo. Sin duda alguna "buenas intuiciones" no suceden al azar: un experto que reflexiona sobre lo que sabe y el origen de lo que sabe, tendrá intuiciones mucho más acertadas que un experto en el tema que no hace estas reflexiones; y en ambos casos, las decisiones serán más atinadas que las de alguien que no es experto en el tema y que, además, no se ha dado a la tarea de reflexionar sobre su propio proceso de toma de decisiones (4). Cabe preguntarnos ¿qué historias han determinado las intuiciones de los equipos interdisciplinarios y a los gobiernos durante la pandemia?
Para los gobiernos, la conformación de estos equipos de apoyo requeriría, por principio, ser una tarea de selección cuidadosa que no esté supeditada a la disposición de los expertos para contribuir voluntariamente. Sociedades que históricamente han implementado modelos de toma de decisiones basados en evidencia tienen un sistema de incentivos que incluye el reconocimiento económico, la confianza, y el reconocimiento público, los que estimula la participación de los científicos en los diferentes sectores de la economía. Aunque este no sea el caso generalizado para América latina, la experiencia de la pandemia ha de servir para seguir creando esta sinergia entre ciencia, gobernanza y sociedad.

Tener un acumulado de evidencia que incluya conocimiento científico, experticia y conocimiento intuitivo, en buena medida determina la calidad de la toma de decisiones, especialmente en estos tiempos. Sin embargo, hay que prestar atención a la subjetividad de quienes toman las decisiones, como por ejemplo, los gobernantes, que durante la pandemia son aún más propensos a la presión social, a la politización y a evaluación constante. Así, pues, llegar a decisiones acertadas no es solo un asunto de los líderes, sino también un asunto social; la sociedad asume su papel validando o no las decisiones tomadas y en tiempos de crisis, los criterios para validar estas decisiones están menos asociados con los hechos y más con las emociones como fuente de información (5).

Al igual que las intuiciones, las emociones son conocimiento a la mano para guiar la toma de decisiones. Son fuente confiable de información en la medida en que se aprenda a rastrear su origen y a calibrar la expresividad de las mismas. Si la voz de la sociedad es la voz de emociones no procesadas, el eco de esta voz puede tener un impacto más negativo que positivo en las decisiones tomadas por los gobiernos.

La susceptibilidad de los gobernantes a la aprobación de la sociedad y a los niveles de popularidad los lleva a escuchar estas voces, no necesariamente porque sean fuente confiable de evidencia, sino porque al hacerlo encuentran una forma de ganar aprobación.

Para contrarrestar este efecto resulta clave que desde la ciencia se tiendan puentes en los que se encuentren diversas voces de varios sectores de nuestra sociedad, y en procura de un diálogo más continuo para que paulatinamente, la ciencia se vaya asentando como una forma cotidiana de ver y leer el mundo. Si en algo puede ayudar la evidencia científica es en generar un diálogo informado e incluyente de las diferentes perspectivas y formas de conocimiento que existen en las sociedades. 
La ciencia ha sido provocadora de conversaciones, de tertulias no necesariamente con rigor científico, pero que han ido dejando historia en el lenguaje, en la manera de interpretar la realidad y de expresar estas interpretaciones. A diferencia de otros países, en los que la ciencia ha hecho parte de la cotidianidad por un buen tiempo, en Colombia y en Latinoamérica esta historia ha comenzado recientemente y la experiencia de la pandemia es una oportunidad para intensificarla y continuarla.

Entre las múltiples huellas que la pandemia pueda dejarnos, está el permitirnos un mejor y mayor acercamiento a la ciencia. Los esfuerzos de algunos gobiernos locales para administrar la pandemia con el apoyo de equipos interdisciplinarios son experiencias de diálogo que bien vale la pena evaluar para dejar a la posteridad, pues enseñan cómo trabajar juntos ciencia, política y sociedad.

La contribución de la ciencia durante la pandemia indudablemente ha ido más allá de contrarrestar los efectos negativos de la enfermedad. La voz de la ciencia no es solamente la voz de los datos. Si bien es cierto que la ciencia y la tecnología han hecho lo propio durante la pandemia, para que este efecto sea largo y duradero es necesario que los gobiernos y la sociedad en general hagan lo suyo en favorecer y mantenerse en diálogo con las diferentes disciplinas, formas y niveles de conocimiento para que la creatividad y la lógica que se combinan en la ciencia sean parte la cotidianidad.

En definitiva, no necesariamente es falta de recursos lo que nos impide satisfacer las necesidades que esta crisis ha generado. Si algo ha impedido el avance en la activación socioeconómica es una necia aspiración a usar los modelos pre-pandemia para una realidad en pandemia. La confianza para sobrepasar esa aspiración no depende de los datos, sino de la creatividad que tengan tanto los gobiernos como las sociedades para reconfigurar el modelo socioeconómico; no para aumentar el consumo, sino para que todos puedan acceder a la oferta. Aunque la ciencia y las tecnologías pueden asistirnos en la toma de decisiones, definir sus criterios y el tipo de buen vivir que buscamos es tarea de nosotros.

(iD Alida María Acosta Ortiz, Psi., MSc., PhD Ciencias cognitivas \& Economía del Comportamiento Facultad de Ciencias de la Salud, Universidad Autónoma de Bucaramanga

\section{Referencias}

1. Vargas-Chaves, I. Una crítica al principio de precaución desde las tensiones sobre su legitimidad y vinculatoriedad. In: Vargas-Chaves, I, Rodríguez, GA, eds. Principio de precaución: desafíos y escenarios de debate. Bogotá: Editorial Universidad del Rosario; 2017. p. $36-67$

2. Insensitivity to future consequences following damage to human prefrontal cortex. Bechara, A.; Damasio, A.R.; Damasio, H. and Anderson, S.W. Cognition. Apr-Jun 1994;50(1-3):7-15. DOI: https://doi.org/10.1016/00100277(94)90018-3

3. Prefrontal Contributions to Metacognition in Perceptual Decision Making. Stephen M. Fleming, Josefien Huijgen and Raymond J. Dolan. Journal of Neuroscience 2 May 2012, 32 (18) 6117-6125. DOI: https://doi.org/10.1523/JNEUROSCI.6489-11.2012

4. The neural system of metacognition accompanying decision-making in the prefrontal cortex. Lirong Qiu , Jie Su, Yinmei Ni , Yang Bai , Xuesong Zhang, Xiaoli Li et al. PLoS Biol, 2018 Apr 23;16(4):e2004037. DOI: https://doi.org/10.1371/journal.pbio.2004037

5. Affective state and decision-making in the Ultimatum Game. Mascha van 't Wout, René S. Kahn, Alan G. Sanfey \& André Aleman. Experimental Brain Research (2006) 169: 564-568. DOI: https://doi.org/10.1007/ s00221-006-0346-5 